\title{
Employing Optical Nonlinearity in Photonic Crystals: A Step Towards All-Optical Logic Gates
}

\author{
Mohammad Danaie and Hassan Kaatuzian \\ Photonics Research Lab., Amirkabir University of Technology \\ Iran
}

\section{Introduction}

Employing nonlinear elements in photonic crystals (PCs) opens up lots of new design opportunities. In comparison to ordinary linear PC structures, using optically nonlinear elements in PCs leads to the observation of many interesting phenomena that can be utilized to design all-optical devices. Optical bistability in PCs is among the mentioned observations, which due to its many applications has attracted the researchers' attention. Many optical devices such as limiters, switches, memories can be implemented when nonlinear elements are embedded in PCs. Kerr type nonlinearity is mostly used for this purpose. In this chapter we are going to discuss the benefits of using nonlinearity in photonic crystal devices. Since most of nonlinear optical devices are either based on directional couplers (DCs) or coupled cavity waveguides (CCWs), we have focused on these two groups to provide a better insight into their future prospects.

The first practical case of employing nonlinearity in PCs was for optical switches. Scholz and his colleagues were among the first ones to use optical Kerr nonlinearity in PCs (Scholz et al., 1998). They designed an all-optical switch using a one dimensional photonic crystal which was placed inside two cross waveguides. In their switch, a strong pump signal lateral to the PC layers made the crystal nonlinear and slightly shifted the position of the bandgap. The data signal wavelength was chosen on the bandgap edge such that it could not be placed in the shifted bandgap region (when the probe signal was present). Therefore; when no probe signal existed, the data signal could not pass through the PC layers, while when the probe was present, the shifted photonic bandgap would allow its transmission. Later the optical bistability in two dimensional nonlinear photonic crystal waveguides coupled to a micro-cavity was discussed in (Centeno \& Felbacq, 2000) and it was suggested that the mentioned phenomena could be use to design all-optical switches. Mingaleev and Kivshar placed nonlinear elements in waveguides and bends to obtain optical limiters (Mingaleev \& Kivshar, 2002). Meanwhile other structures were suggested by (Soljac`ic' et al., 2002) and (Fan, 2002) for optical switching. Later in 2004 (Locatelli et al., 2004) and (Cuesta-Soto et al, 2004) suggested optical switches using directional couplers. Thereafter, some logic components were reported in literature such as: An optical AND gate by (Zhu et al., 2006), an all-optical PC on-chip memory implemented in (Shinya et. al., 2008), a PC half-adder structure designed in (Liu et al., 2008) and so forth. 
In this chapter, first different types of optical nonlinearities are briefly explained in electromagnetics' terms. Thereafter, numerical and analytic methods for modeling optical nonlinearity in photonic crystals are reviewed. As a case study nonlinear optical Kerr effect is used to design a directional coupler switch and later as the second case study an structure for all-optical AND gate operation is proposed.

\section{Optical nonlinearity in PCs}

Nonlinear optics field came to existence when (Franken et al., 1961) published their achievements on second harmonic generation. It was mainly due to acquiring the technology to produce high intensity coherent lights that could trigger the nonlinearity in materials. Such ability was facilitated by the introduction of laser by (Maiman, 1960). Since then many other optical phenomena such as parametric oscillation, four wave mixing, stimulated Raman and Brillouin scattering, phase conjugation etc. have been observed and studied (Boyd, 2003).

Nonlinear optical materials can be considered as the basic elements for all-optical processing systems. Although optical bistability in nonlinear materials has been extensively studied since 1980 (Saleh, 1991 and Kaatuzian, 2008), but since most materials exhibit pretty weak nonlinear characteristics, the large size and high operational power of such devices makes them unsuitable for all-optical integrated devices. Introduction of PCs, due to its unique characteristics (Yablonovitch, 1987 and John, 1987), helped to reduce both the operational power and size of nonlinear optical components and presented a new opportunity for their integration.

In a linear material the dipole moment density (or equally known as polarization) $P(t)$ and electric field $\mathrm{E}(\mathrm{t})$ have a linear relationship:

$$
P(t)=\varepsilon_{0} \chi E(t)
$$

The notation " $\chi$ " is usually referred to as susceptibility. In a nonlinear medium (1) can be generalized as follows (Boyd, 2003):

$$
\begin{aligned}
& P(t)=\varepsilon_{0}\left[\chi^{(1)} E(t)+\chi^{(2)} E^{2}(t)+\chi^{(3)} E^{3}(t)+\ldots\right] \\
& \quad=P^{1}(t)+P^{2}(t)+P^{3}(t)+\ldots \\
& =P^{L}+P^{N L}
\end{aligned}
$$

where, the notations $\chi^{(1)}, \chi^{(2)}, \chi^{(3)}$ are called the first, second and third order susceptibility coefficients respectively. Also $\mathrm{P}^{1}(\mathrm{t}), \mathrm{P}^{2}(\mathrm{t}), \mathrm{P}^{3}(\mathrm{t})$ are referred to as the first, second and third order polarizations. Therefore, $\mathrm{P}(\mathrm{t})$ can be considered to have a linear term $\mathrm{PL}$ and a nonlinear term PNL. In nonlinear optics, most of nonlinear observations are usually due to $\chi^{(2)}$ and $\chi^{(3)}$ which is mainly due to the fact that the higher order terms need much higher input intensities to show significant impact. In addition it is shown (Boyd, 2003) that $\chi^{(2)}$ can be only observed in non-centrosymmetric crystals; therefore liquids, gas and many solid crystals do not display those phenomena that are originated by $\chi^{(2)}$.

The $i^{\text {th }}$ component for the vector P can be expressed as (3) (Saleh, 1991): 


$$
P_{i}=\varepsilon_{0} \sum_{j=1}^{3} \chi_{i j}^{(1)} E_{j}+\varepsilon_{0} \sum_{j=1}^{3} \sum_{k=1}^{3} \chi_{i j k}^{(2)} E_{j} E_{k}+\varepsilon_{0} \sum_{j=1}^{3} \sum_{k=1}^{3} \sum_{l=1}^{3} \chi_{i j k l}^{(3)} E_{j} E_{k} E_{l}+\ldots,
$$

where, $i=1,2,3$ (corresponding to $x, y, z$ ). For a linear material, only the first term of this equation is significant. One of the most important nonlinear optical effects which originates from the $\chi^{(3)}$ coefficients is the Kerr effect (Weinberger, 2008). It was discovered in 1875 by John Kerr. Almost all materials show a Kerr effect. The Kerr effect is a change in the refractive index of a material in response to an external electric field. For materials that have a non-negligible Kerr effect, the third, $\chi(3)$ term is significant, with the even-order terms typically dropping out due to inversion symmetry of the Kerr medium. In the optical Kerr effect, an intense beam of light in a medium can itself provide the modulating electric field, without the need for an external field to be applied. In this case, the electric field is given by:

$$
E=E_{\omega} \operatorname{Cos}(\omega t) \text {. }
$$

where $E_{\omega}$ is the amplitude of the wave. Combining this with the equation for the polarization will yield:

$$
P \simeq \varepsilon_{0}\left(\chi^{(1)}+\frac{3}{4} \chi^{(3)}\left|E_{\omega}\right|^{2}\right) E_{\omega} \operatorname{Cos}(\omega t)
$$

As before:

$$
\chi=\chi^{L}+\chi^{N L}=\chi^{(1)}+\frac{3}{4} \chi^{(3)}\left|E_{\omega}\right|^{2}
$$

and since:

$$
n=\sqrt{1+\chi}=\sqrt{1+\chi^{L}+\chi^{N L}} \simeq n_{0}\left(1+\frac{1}{2 n_{0}^{2}} \chi^{N L}\right),
$$

where $\mathrm{n}_{0}=\left(1+\chi^{\mathrm{L}}\right)^{1 / 2}$ is the linear refractive index. Therefore:

$$
n=n_{0}+\frac{3 \chi^{(3)}}{8 n_{0}}\left|E_{\omega}\right|^{2}=n_{0}+n_{2} I
$$

where $\mathrm{n}_{2}$ is the second-order nonlinear refractive index, and $\mathrm{I}$ is the intensity of the wave. The refractive index change is thus proportional to the intensity of the light travelling through the medium.

\subsection{Modeling optical nonlinearity in PCs}

In order to numerically analyze linear PCs, various methods have been proposed in the literature. Among these methods, the most popular are: plane wave expansion (PWE), finite difference in time domain (FDTD), finite element method (FEM), multiple multimode method (MMP) and Wannier function method (WFM). In the nonlinear regime, in addition to the mentioned methods, complementary strategies have to be used to analyze the effect of the 
nonlinear elements on the linear system. Transfer matrix method (TMM), perturbation theory, coupled mode theory (CMT) and FDTD can be used for this purpose. In order to model the Kerr effect using FDTD, several methods have been proposed. Here FDTD and perturbation theory are briefly reviewed. Also In section 4 CMT is used to analyze a PC limiter.

From the Maxwell equations, the wave equation for the nonlinear medium can be written as:

$$
\nabla^{2} E-\mu_{0} \varepsilon_{0} \varepsilon_{r}(x, y, z, \omega, E) \frac{\partial^{2} E}{\partial t^{2}}=0 .
$$

It is shown in (Joseph \& A Taflov, 1997) that (9) can be rewritten as:

$$
\nabla^{2} E-\mu_{0} \varepsilon_{0} n_{0}^{2} \frac{\partial^{2} E}{\partial t^{2}}=\mu_{0} \frac{\partial^{2} P^{N L}}{\partial t^{2}}
$$

Based on the type of nonlinearity different approaches have to be made to solve the mentioned equation. The FDTD model for Kerr-type materials assumes an instantaneous nonlinear response. The nonlinearity is modelled in the relation $D=\varepsilon E$ where:

$$
\varepsilon=n^{2}=\left(n_{0}+\frac{3 \chi^{(3)}}{8 n_{0}}\left|E_{\omega}\right|^{2}\right)^{2} \simeq n_{0}{ }^{2}+2 n_{0} \frac{3 \chi^{(3)}}{8 n_{0}}|E|^{2} .
$$

Therefore the relation ship between $\mathrm{E}$ and $\mathrm{D}$ can be iteratively determined using:

$$
E=\frac{D}{n_{0}^{2}+\frac{3 \chi^{(3)}}{4}|E|^{2}} .
$$

Perturbation theory is also a useful tool in engineering for analyzing systems with small nonlinearities. Using Maxwell equations, the following eigenvalue problem can be derived for linear time invariant PC systems (A waveguide is assumed in our case.). The Dirac notation $\left|\vec{E}_{0}\right\rangle$ specifies the Bloch eigenmode for the electric field.

$$
\nabla \times \nabla \times\left|\vec{E}_{0}\right\rangle=\left(\frac{\omega_{0}}{c}\right)^{2} \varepsilon_{0}(\vec{r})\left|\vec{E}_{0}\right\rangle .
$$

It is shown in (Bravo-Abad et al., 2007) that a $\Delta \varepsilon$ change in the dielectric constant can result in a $\Delta \omega$ variation in the original eigenvalue $\omega_{0}$ as:

$$
\Delta \omega=-\frac{\omega_{0}}{2} \frac{\left\langle\vec{E}_{0}|\Delta \varepsilon| \vec{E}_{0}\right\rangle}{\left\langle\vec{E}_{0}\left|\varepsilon_{0}\right| \vec{E}_{0}\right\rangle}=-\frac{\omega_{0}}{2} \frac{\int d^{3} r \Delta \varepsilon(\vec{r})\left|\vec{E}_{0}(\vec{r})\right|^{2}}{\int d^{3} r \varepsilon_{0}(\vec{r})\left|\vec{E}_{0}(\vec{r})\right|^{2}} .
$$

But since for a waveguide $v_{g} \approx \Delta \omega / \Delta k$ then it can be shown that (Bravo-Abad et al., 2007) the following approximation is valid: 


$$
\Delta k=-\frac{\omega_{0}}{v_{g}} \frac{\Delta n}{n_{r}} \frac{\int_{A L G H-\varepsilon} d^{3} r \varepsilon_{0}(\vec{r})\left|\vec{E}_{0}(\vec{r})\right|^{2}}{\int_{A L S P A C E} d^{3} r \varepsilon_{0}(\vec{r})\left|\vec{E}_{0}(\vec{r})\right|^{2}} .
$$

The mentioned change in the wavenumber can cause an optical phase difference between the linear and nonlinear states of a system. Many optical devices such as directional couplers or Mach-Zehnder interferometers are sensitive to the induced phase. Assuming that $\mathrm{L}$ is the waveguide length, $\Delta \phi=L \Delta k$ shows the phase difference between the linear and nonlinear cases. According to (15) if the waveguide is designed to have a low group velocity, for a fixed amount of refractive index variation a larger $\Delta k$ is obtained. It means that for a fixed $\Delta \phi$ a smaller device size L is needed; or equally for a fixed L a smaller $\Delta n$ (which is proportional to operational power) is required. Since photonic crystals can be used to design waveguides with very low group velocities (Solja 'cic' \& Joannopoulos, 2004); therefore they provide the opportunity to reduce both the device size and the operational power; making such devices able to be integrated on a single chip.

\section{Case study 1: A PC all-optical switch}

The idea of a one dimensional PC all-optical switch was first proposed in (Scholz et al., 1998). Coupled cavity waveguides (CCW) with Kerr nonlinearity, were there after suggested for all-optical switching. The main drawback of CCW switches is that when the switch is in the OFF state, all the data signal is reflected back to the input port. Since the backscattered signal can affect other optical devices on an optical chip, it makes them unsuitable for all optical integrated circuit applications. A combination of directional couplers and nonlinear optical elements can be used to solve the mentioned problem (Yamamoto et al., 2006, Cuesta-Soto et al., 2004, Rahmati \& N. Granpayeh, 2009).

In a directional coupler based switch, according to the ON or OFF state of the switch, most of the data signal power is guided to either of the two output ports. Usually only a very small amount leaks to the input port. It gives the designer the ability of using the switch in sequential optical circuits.

Here a PC directional coupler is designed first.The PC lattice used for this design is a two dimensional array of GaAs rods which is known to have Kerr type optical nonlinearity.

The band diagram of the PC, Which is obtained using Plane Wave Expansion (PWE) method, is shown in Fig. 1. The shaded region is the optical bandgap. No optical signal within the normalized frequency range of $0.28 \mathrm{a} / \lambda$ to $0.45 \mathrm{a} / \lambda$; where $\mathrm{a}$ is the lattice constant and $\lambda$ is free space wavelength, can propagate through the PC lattice. The value of a is chosen $635 \mathrm{~nm}$ in our simulations. The radii of rods are equal to $0.2 \mathrm{a}$ and their refractive index is chosen equal to 3.4, which is equal to the refractive index of GaAs at the $1550 \mathrm{~nm}$ wavelength.

Introduction of defects into PC lattice is the first step in designing PC devices. These are usually classified in two different categories of point defects and line defects, which can create resonators and waveguides respectively. As an Instance, in the mention PC, removing a column of rods (Fig. 2a) creates a waveguide mode in the bandgap region between the 
normalized frequencies $0.340 \mathrm{a} / \lambda$ and $0.447 \mathrm{a} / \lambda$ (See Fig. 3a). A simple directional coupler can be obtained by removing two rows of rods adjacent to a central row (See Fig. 2b.) (Zimmermann et al., 2004, Nagpal, 2004). As discussed in (Nagpal, 2004), when two PC waveguides are placed close to each other, light propagating in one of the waveguides can be coupled to the neighboring waveguide.

TM Band Structure

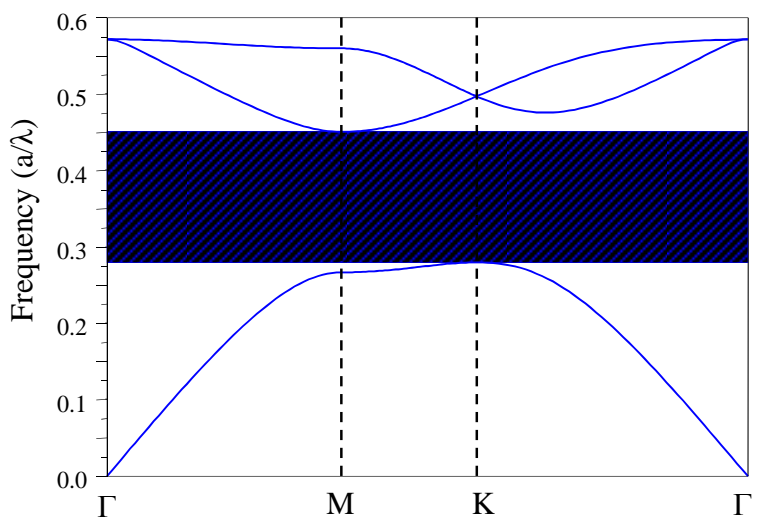

Fig. 1. The band diagram for a 2D hexagonal array of GaAs rods, where the ratio of the rods radius to the lattice constant is 0.2 .

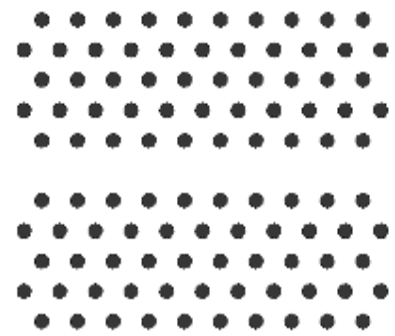

(a)

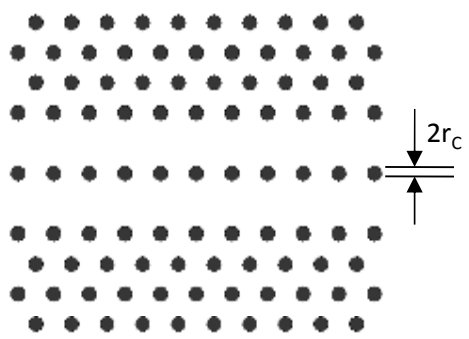

(b)

Fig. 2. Different PC structures for TM modes: The dark circles are assumed to be dielectric and the background is air (a) a PC W1 waveguide (b) A PC directional coupler.

PWE method can also be used to analyze the structure in Fig. 2b. In this case, using a supercell, the two adjacent waveguides can be treated as a single symmetric line defect in the PC structure. The super-cell which is used for this purpose and the band diagram obtained in this case are shown is Fig. 3b. Here, the radii of the central rods, $r_{C}$, is assumed 0.2a. An odd and an even defect mode are produced in this case.

It is shown (Zimmermann et al., 2004, Nagpal, 2004)] that the light that is travelling in one of these waveguides, can be periodically coupled to the other one after passing a certain distance referred to as the coupling length $\left(\mathrm{L}_{\mathrm{C}}\right)$. The coupling length is related to the propagation constants of the odd (ko) and even (ke) modes as follows: 


$$
L_{C}=\frac{\pi}{k e-k o} \text {. }
$$

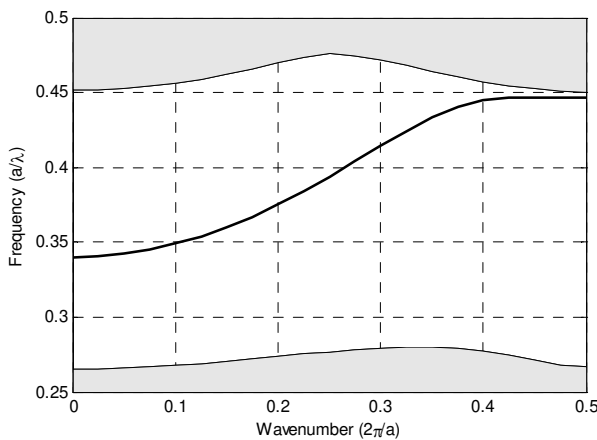

(a)

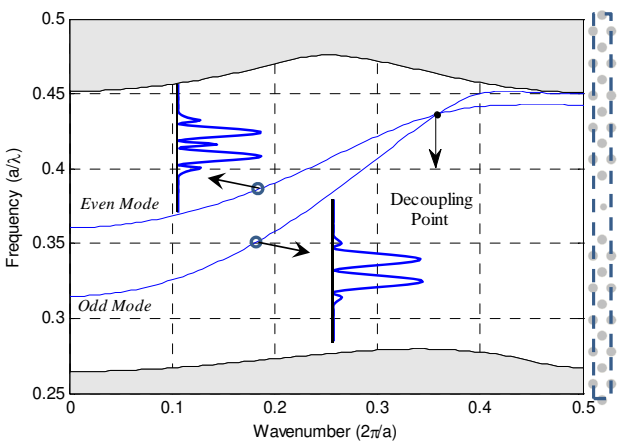

(b)

Fig. 3. The waveguide modes for (a) the W1 waveguide shown in Fig. 2(a) and (b) the directional coupler shown in Fig. 2(b). The super-cell for obtaining the directional coupler is depicted in the dashed box.
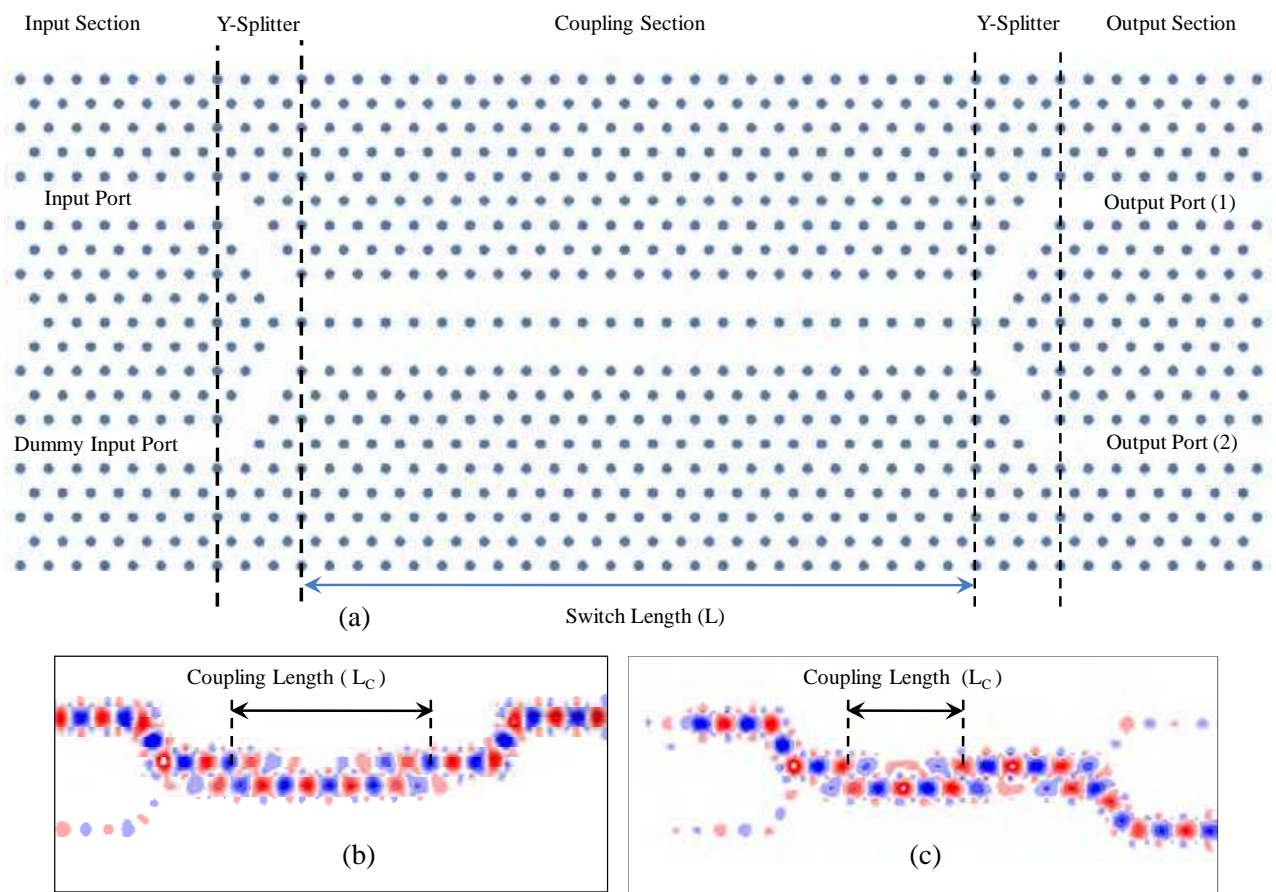

Fig. 4. (a) A conventional PC directional coupler (b) $f=410 a / \lambda$ (c) $f=0.396 a / \lambda$. 
Directional couplers can be used as wavelength selective devices, in their linear regime, or switches when optical nonlinearity is introduced to their structure. A typical symmetrical directional coupler switch is shown in Fig. 4a. It consists of two input ports, two output ports and a central coupler. Assuming that a signal has entered from one of the input ports, it passes through the bends and enters the coupling region which has a length equal to $\mathrm{L}$ (several times larger than $\mathrm{L}_{\mathrm{C}}$ ). The signal is then periodically transferred between the two waveguides and regarding the ratio between $\mathrm{L}$ and $\mathrm{L}_{\mathrm{C}}$, it will be directed to either one of the output ports (or even both for a poor design).

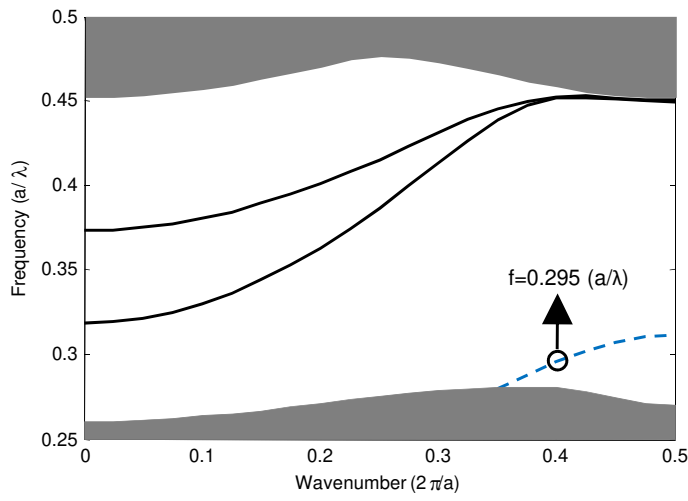

(a)

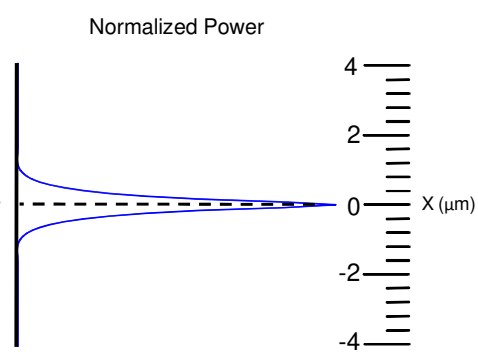

(b)

Fig. 5. (a) The band diagram for the directional coupler shown in Fig. 2(b) when $r_{C}$ is reduced to $0.15 \mathrm{a}$, (b) The spatial profile for the control mode at $\mathrm{f}=0.295(\mathrm{a} / \lambda)$.

Fig. $4 \mathrm{~b}$ and Fig.4c demonstrate the selective behavior of the device for two different input wavelengths. Since from (16) the coupling length is inversely related to the difference of ke and ko; then according to Fig. $3 b$, it is obvious that for each input signal frequency a different coupling length is perceived. This phenomenon can be used to separate different wavelengths (Fig. 4b and Fig. 4c).

Reduction of $\mathrm{r}_{\mathrm{C}}$ create a mode that can be used as the control signal. Fig. 5a shows the band diagram for the directional coupler in Fig. $2 b$ when $r_{C}$ is $0.15(a / \lambda)$. As seen in Fig. $5 b$ the mentioned mode is highly confined. Since the rods are nonlinear, if the control signal power is large enough the refractive index of the middle row can be changed which will affect the system behaviour in regard to the linear case.

In a conventional directional coupler, if the refractive index of the central region between the two waveguides is somehow changed such as: $n \rightarrow n+\delta n$, then the wavenumber of the odd and even modes change accordingly to ke $+\delta$ ke and ko $+\delta$ ko. From (16), it is obvious that the new coupling length for the modified structure will be slightly different. Assuming that the difference in the coupling length be $\delta L_{C}$, and that $m=L / L_{c}$, then if $(m+1) \delta L_{c}$ equals a coupling length, the light will be transferred to the other output port. It means that a change in the refractive index of the central row can provide a switching mechanism. From the previous equations, it can be concluded that $(m+1) \delta L_{C}=L_{C}$ is the switching condition. Since $\mathrm{m}=\mathrm{L} / \mathrm{L}_{\mathrm{C}}$ then: 


$$
L=L_{C}^{2} \times \frac{1}{\delta L_{c}}-L_{C}
$$

Also:

$$
\delta L_{C}=\pi\left(\frac{1}{k e-k o}-\frac{1}{k e+\delta k e-k o-\delta k o}\right) \cong \pi \frac{\delta k e-\delta k o}{(k e-k o)^{2}} .
$$

Neglecting the $\mathrm{L}_{\mathrm{C}}$ term with regard to $\mathrm{L}^{2} \mathrm{C} / \delta \mathrm{L}_{\mathrm{C}}$ in (17) leads to:

$$
L \cong L_{C}^{2} \times \frac{(k e-k o)^{2}}{\pi\left(\delta k_{e}-\delta k_{o}\right)}=L_{C}^{2} \times \frac{(k e-k o)^{2}}{\pi \delta k} .
$$

Using (16), equation (19) can be simplified to:

$$
L \cong \pi \times \frac{1}{\delta k} .
$$

According to (20), the switch length is inversely proportional to $\delta k$; which is itself a function of $\delta \mathrm{n}$. The relationship between $\delta \mathrm{k}$ and $\delta \mathrm{n}$ is dependant upon the structure geometry and is usually determined numerically using PWE method. In order to minimize the switch length and operational power, it is very important that for a slight variation in $n$, a large $\delta \mathrm{k}$ be obtained. In (Yamamoto et al., 2006) some methods are suggested to improve the $\delta \mathrm{k}$ and $\delta \mathrm{n}$ relation for a PC directional coupler for TE modes.

The refractive index can be modified using electro-optic, thermo-optic or Kerr optical effect. From the mentioned optical phenomena, only Kerr effect can be used for all-optical applications. In materials that possess the Kerr effect, the refractive index can be linearly changed using an optical pump signal as follows:

$$
n=n_{0}+n_{2} I
$$

where, $\mathrm{n}_{0}$ is the refractive index in the linear regime, $\mathrm{I}$ is the optical field intensity and $\mathrm{n}_{2}$ is the nonlinear Kerr coefficient. Since $\delta k$ is a function of $\delta n$, it can be seen from (20) that there is a trade-off between switch size and power, i.e. in order to reduce the operating power of the switch, longer device size is needed, or vice versa. The relationship between $\delta \mathrm{k}$ and $\delta \mathrm{n}$ should be calculated numerically (Danaie \& Kaatuzian, 2011). Afterwards according to (20) the required switch length can be estimated for different signal frequencies. PWE method can be used for this purpose. We have depicted the relationship between signal frequency $\left(f_{s}\right)$ and $\delta k$ in Fig. 6.

In order to be able to optimize the switch length, we first try to fit an analytical expression on the curves shown in Fig. 6. Each of the five curves in Fig. 6 can be considered to be the bottom black curve multiplied by a $\gamma$ factor. If $\gamma$ is calculated for the four upper curves, it is seen that these factors are neatly placed on a line. On the other hand, the first glance at the curves shown in Fig. 6 suggests an exponential relation between $\delta \mathrm{k}$ and frequency; while a more accurate study reveals second order polynomial relation. 


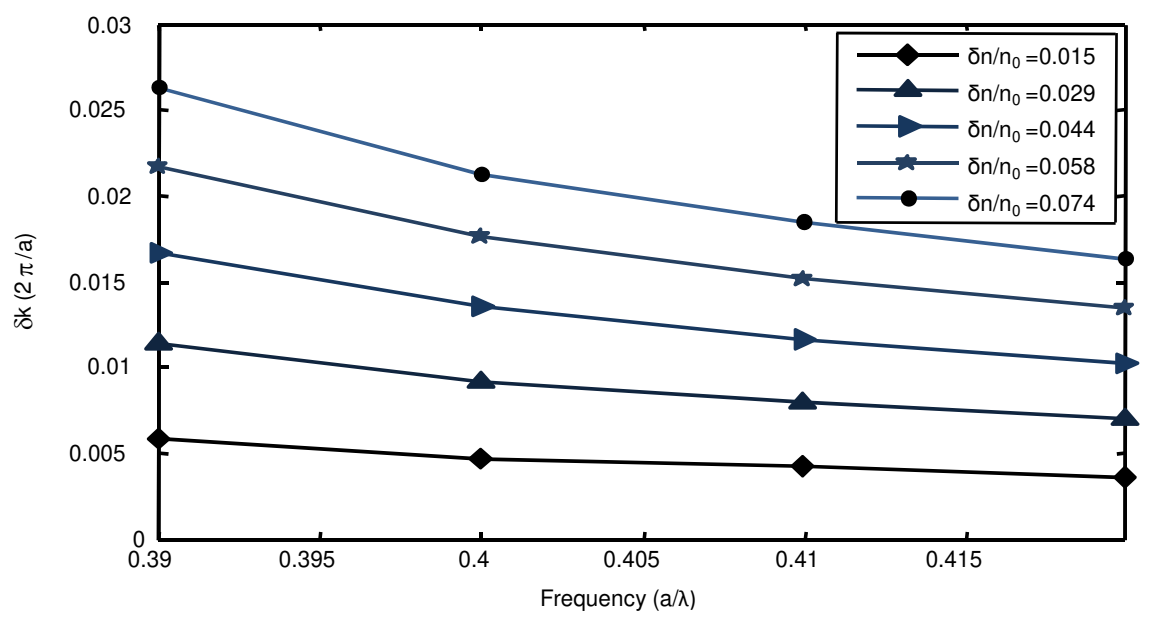

Fig. 6. The relationship between signal frequency and $\delta \mathrm{k}$ of the directional coupler depicted in Fig. 2(b) with $r_{C}=0.15 a$, obtained using PWE method for different values of central row's refractive index change.

Based on the previous observations the relationship between $\delta \mathrm{k}$ and $\delta \mathrm{n}$ can be assumed as:

$$
\delta k\left(\delta n, f_{s}\right)=h(\delta n) \cdot g\left(f_{s}\right),
$$

where $f_{s}$ is the signal frequency and $h$ and $g$ are first and second order polynomials dertermined as:

$$
\begin{gathered}
h(\delta n)=a \cdot \delta n+b . \\
g(f)=c \cdot f_{s}^{2}+d \cdot f_{s}+e .
\end{gathered}
$$

For our structure $\left(\mathrm{r}_{\mathrm{C}}=0.15 \mathrm{a}\right)$, the factors $a-f$ are determined as follows using curve fitting techniques:

$$
\delta k(\delta n, f)=(17.5 \delta n+0.17) \cdot\left(1.25 f_{s}^{2}-1.08 f_{s}+0.24\right)
$$

The previous equation can be used for optimization purpose; in which the $f$ is considered per $(a / \lambda)$ and the $\delta k$ is per $(2 \Pi / a)$. Since $\delta n=n_{2} I$, then the relationship between the switch length (per lattice constant) and optical power intensity (on the rods) becomes as follows:

$$
L \cong \pi \times \frac{1}{\left(\frac{2 \pi}{\mathrm{a}}\right)(a \cdot \delta n+b)\left(c \cdot f_{s}^{2}+d \cdot f_{s}+e\right)}=\frac{\mathrm{a}}{2\left(a \cdot n_{2} I+b\right)\left(c \cdot f_{s}^{2}+d \cdot f_{s}+e\right)} .
$$

In the above equation a is the latice constant. If the designer wishes to choose the input signal for the $1550 \mathrm{~nm}$ wavelength, then the the lattice constant must be chosen equal to $\mathrm{f}_{\mathrm{s}} .1550 \mathrm{~nm}$; therefore the actual size of the switch becomes equal to: 


$$
L \cong \frac{f_{s} \cdot 1550 \mathrm{~nm}}{2\left(a \cdot n_{2} I+b\right)\left(c \cdot f_{s}^{2}+d \cdot f_{s}+e\right)} .
$$

When an optical pulse enters a medium with a low group velocity, it becomes squeezed in the time domain. In order for the pulse energy to remain constant its amplitude should increase (Solja 'cic' \& Joannopoulos, 2004). The term $\mathrm{n}_{2} \mathrm{I}$ in (25) can be therefore rewritten as a. $n_{2} . \mathrm{I}_{\text {in }}\left(\mathrm{c}_{0} / \mathrm{vg}\right)$; where $\mathrm{I}_{\text {in }}$ is the input power intensity of the control signal; $\mathrm{v}_{\mathrm{g}}$ is the group velocity, $\mathrm{c}_{0}$ is the free space light speed and $a$ is a correction factor which is related to the control signal mode profile. It determines what ratio of the input power is concentrated on the nonlinear rods. The a factor should be calculated numerically for each frequency. Since $v_{g}$ and $\alpha$ are functions of the control frequency $\left(f_{c}\right)$, therefore equation (25) is then rewritten as:

$$
L \cong \frac{f_{s} \cdot 1550 \mathrm{~nm}}{2\left(a \cdot n_{2} I_{i n}\left(\frac{c_{0}}{v_{g}\left(f_{c}\right)}\right) \alpha\left(f_{c}\right)+b\right)\left(c \cdot f_{s}^{2}+d \cdot f_{s}+e\right)} .
$$

The relation between the group velocity and $f_{c}$ can be obtained using PWE. For $f_{c}=0.295$ $(\mathrm{a} / \lambda)$ a can be obtained from integration of control mode profile from $\mathrm{x}=-95 \mathrm{~nm}$ to $+95 \mathrm{~nm}$ (the central rods section). Using a simple integration it can be seen that 0.35 percent of the control power is located on the rods (Fig. 5b).

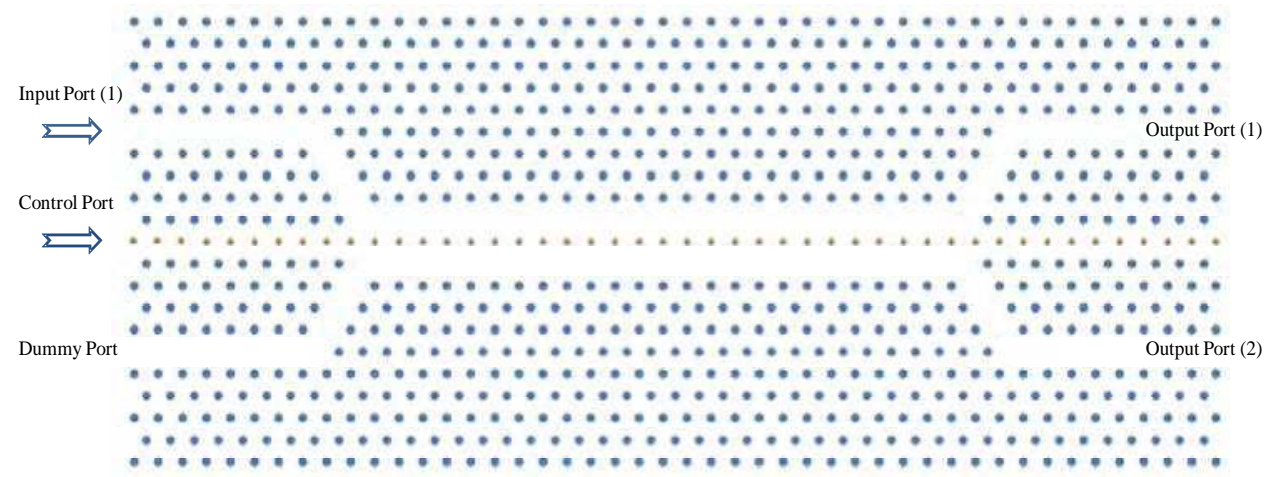

Fig. 7. The all-optical switch designed using a directional coupler.

As a case study, we have designed a switch in this section. The control signal frequency is chosen $f_{c}=0.295(a / \lambda)$, so as to minimum the reflection in the control signal path. The signal frequency is chosen $f_{s}=0.41(a / \lambda)$. Since:

$$
L \cong \frac{\mathrm{a}}{2(17.5 \delta n+0.17) \cdot\left(1.25 f_{s}^{2}-1.08 f_{s}+0.24\right)}
$$

then if we decide the switch length $L$ to be equal to $25 \mathrm{a}$, then according to (27) the required Sn will be equal to 0.204 . We use 2D FDTD method to analyze the time domain behavior of the directional-coupler-based switch. The rods are assumed to have Kerr type nonlinearity 
and the nonlinear Kerr coefficient $\mathrm{n}_{2}$ is assumed $1.5 \times 10^{-17} \mathrm{~m}^{2} / \mathrm{W}$. Each unit cell is meshed $16 \times 16$. The final switch structure is shown in Fig. 7 .

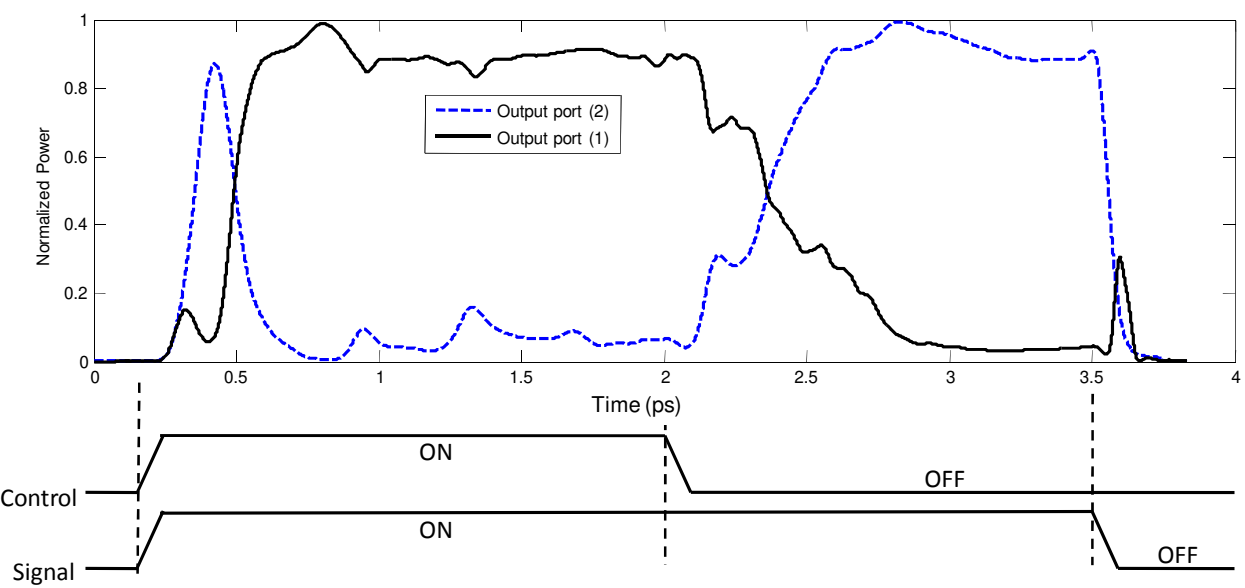

Fig. 8. FDTD time domain simulation results for the directional coupler shown in Fig. 7. The solid line represents the optical power directed to the first output port and the dashed line shows the optical power which goes to the second output port.

In order to be able to observe the time-domain characteristics of the switch, the control and signal inputs are turned on simultaneously. After $2 \mathrm{ps}$ the control signal is turned off and after 3.5ps the data signal is turned off as well. Fig. 8 show the power density versus time for the first and second output ports for the structure shown in Fig. 7. Since the group velocity of the data signal is higher than the control signal, it reaches the output section faster. Thus for a brief period of time $(t=0.4 \mathrm{ps})$, it goes to the second output port. At $t=0.5 p s$ the control signal reaches the output section (turns the rods nonlinear) and forces the data signal to travel to the first output port. When the control signal is turned off $(t=2 p s)$, the rods are tuned linear again and the data signal travels back to the first output port.

\section{Case study 2: Design of an optical gate using CCWs}

Recently more research attention has been directed towards photonic crystal logic gates (Andalib \& Granpayeh, 2009, Bai eta al., 2009). In (Zhu et al., 2006) a structures for Alloptical AND gate is proposed. The main problem of the structures is that the AND gate's inputs have different input wavelengths. In an ideal two-input AND gate, the wavelength of the inputs should be the same or the mentioned AND gate cannot be used in all-optical large-scale circuits. Here a photonic crystal AND gate with identical input characteristics is proposed. Most PC optical devices reported in the literature use TM photonic crystal structures, such as a square lattice of dielectric rods to create a switching mechanism; while here a triangular lattice of holes in a dielectric substrate is used instead (which can be used for TE modes). It can provide a considerably large bandgap and can easily be fabricated using integrated circuit manufacturing technology. The radius of the holes is chosen 0.3a. Choosing larger holes will reduce the guiding strength in vertical direction. The dielectric is 
assumed to be GaAs as before. In this section, first a sharp PC limiter is designed and then by combining it with a Y-junction, a PC gate is obtained.

\subsection{PC limiters}

One of the best methods to be able to observe a strong switching mechanism in a PC is to create a nonlinear PC cavity that is coupled to a PC waveguide. Optical nonlinearity can shift the position of the cavity, which can result in creating a hysteresis loop in the path of signal (i.e. an optical limiter can be created.). In order to do so, the PC structure, waveguide topology and cavity specifications each play an important role.
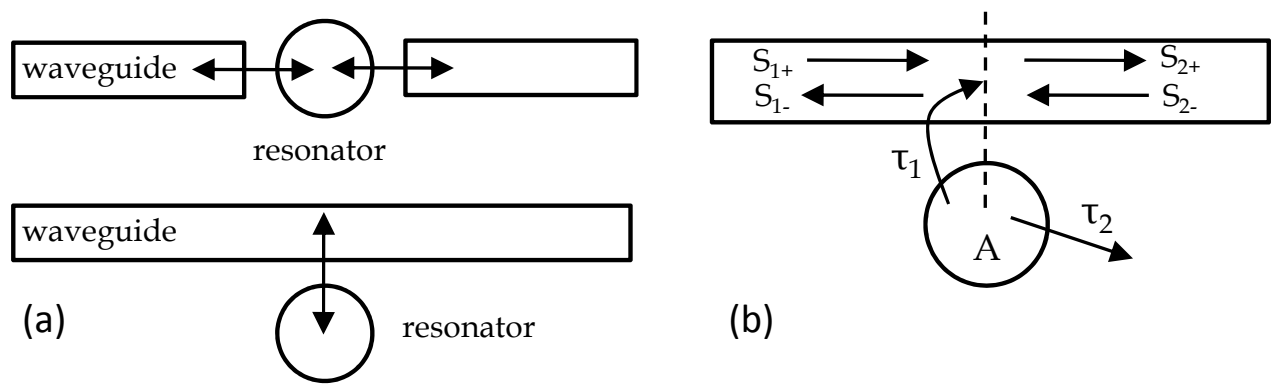

(b)

Fig. 9. (a) Two different possible coupling schemes between a cavity and a waveguide (b)

Schematic description of CMT model parameters for the side coupled cavity waveguide.

It was briefly mentioned that FDTD and perturbation methods can be used to analyze PCs which have optical nonlinearity. In this section another popular method is used to explain the behaviour of a nonlinear PC component. CMT is a general method that can be used for analyzing the behaviour of cavities that are weakly coupled to a waveguide (Saleh, 1991). Generally a cavity can be either placed at the end of a waveguide (directly coupled) or be side-coupled to one (Yanik et al., 2003) (See Fig. 9a).

In the linear case, if we assume that the EM fields inside the cavity are proportional to the parameter A (Fig. 9b), this system can be modelled using CMT by the following set of equations (Bravo-Abad et. al, 2007):

$$
\begin{aligned}
\frac{d A}{d t} & =i \omega_{c} A-\frac{1}{\tau_{1}} A-\frac{1}{\tau_{2}} A+\kappa s_{1+}+\kappa s_{2-} \\
s_{1-} & =s_{2-}-\kappa A \\
s_{2+} & =s_{1+}-\kappa A,
\end{aligned}
$$

where $\omega_{c}$ is the resonant frequency of the cavity. $\tau_{1}$ is the decay constant of the cavity into the waveguide modes propagating to the right and to the left; while the magnitude $\tau_{2}$ relates to the decay rate due to cavity losses. The parameters $\mathrm{s}_{1+}$ and $\mathrm{s}_{1-}\left(\mathrm{s}_{2+}\right.$ and $\left.\mathrm{s}_{2-}\right)$ denote the complex amplitudes of the fields propagating to the left and right at the input (output) of the waveguide. The parameter $\mathrm{k}$ governs the input coupling between the resonant cavity and the propagating modes inside the waveguide. From (Bravo-Abad et. al, 2007) this magnitude is given by $\mathrm{\kappa}=\left(1 / \tau_{1}\right)^{(1 / 2)}$. 
Now, if we assume that the input signal launched only from the left $\left(\mathrm{s}_{2}=0\right)$ and that external losses can be neglected $\left(\tau_{2} \rightarrow \infty\right)$, (29) can be expressed in terms of just $\mathrm{s}_{1+}$ and $\mathrm{s}_{1-}$ as:

$$
\frac{d s_{1-}}{d t}=i \omega_{c} s_{1-}-\frac{1}{\tau_{1}} s_{1-}-\frac{1}{\tau_{1}} s_{1+} .
$$

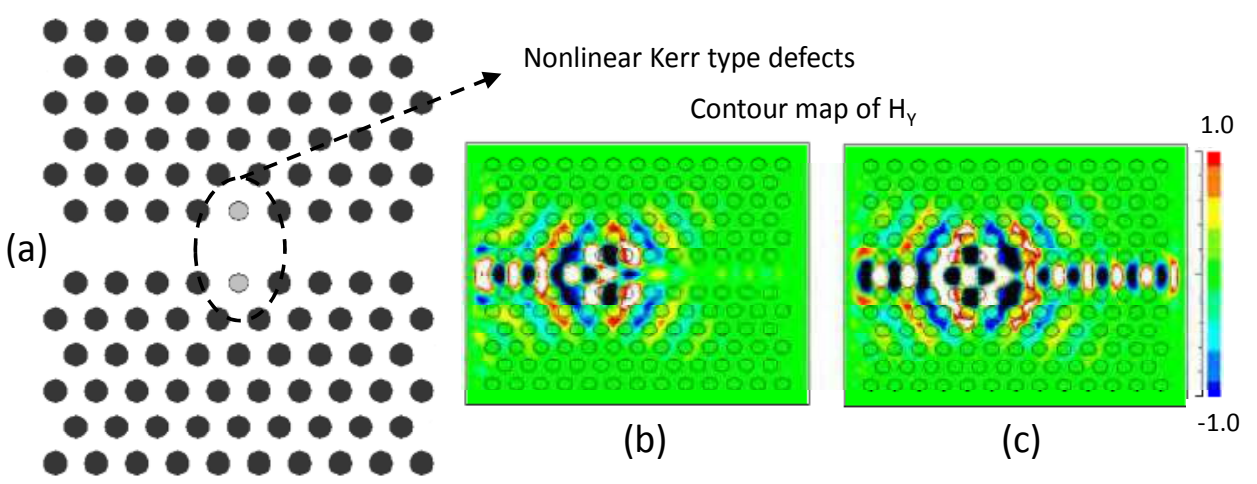

Fig. 10. (a) A simple symmetric side-coupled cavity waveguide. The dark circles are assumed to be air holes in a GaAs substrate and the grey circles are assumed to contain the nonlinear elements. (b) Steady state response of the limiter for the linear case i.e. low input intensity (c) Transmission for high input intensity.

It can be shown that for Kerr type nonlinearity:

$$
\frac{d s_{1-}}{d t}=i \omega_{c}\left(1-\frac{1}{2 Q} \frac{\left|s_{1-}\right|^{2}}{P_{0}}\right) s_{1-}-\frac{1}{\tau_{1}} s_{1-}-\frac{1}{\tau_{1}} s_{1+},
$$

where $Q$ is the quality factor of the cavity and $P_{0}$ is characteristic power of the system (See (Bravo-Abad et. al, 2007) for its definition.).

The PC used in this section to create a waveguide is a triangular lattice of holes in a GaAs substrate. The radius of the holes is chosen 0.3a. The triangular lattice of air holes can be more easily fabricated than the rod lattice version which is used for TM modes. To create a waveguide a row of holes is removed from the PC.

In order to create a cavity mode in the bandgap region of a PC, a defect has to be added to the structure of the waveguide. The defect can later be doped with a material which exhibits strong Kerr effect (Fushman et al., 2008, Nakamura et al., 2004) so as to be able to create the desirable hysteresis effect. Doping quantum dots in to photonic crystals has long been known to create large Kerr type nonlinearity coefficients. Here, the defect is assumed to be doped using the method presented in (Nakamura et al., 2004) resulting in a refractive index equal to 2.6 and a nonlinear Kerr effect equal to $\mathrm{n}_{2}=2.7 \times 10^{-9} \mathrm{~m}^{2} / \mathrm{W}$. The defect radius is $r_{d}=0.25 \mathrm{a}$. First the structure shown in Fig. 10a is chosen as the limiter. The grey circles are assumed to be the nonlinear elements. FDTD method is used for time domain analysis of 
such a device. First we assume that the PC device is comprised of linear element $\left(\mathrm{n}_{2}=0\right)$; in such a case the input frequency is tuned at the cavity's notch. No signal can pass the waveguide in this case. Then we assume that the defect has Kerr effect which causes a shift in cavity's central frequency and allows the signal to pass. The switching mechanism of this limiter is shown in Fig. 10b and Fig. 10c.

The mentioned structure cannot provide a sharp limiting operation. Based on our observations, if a number of cavities are used in cascade a sharper curve can be obtained. It should be noted that the distance between the cavities should be chosen long enough so that they cannot have loading effect on each other. The proposed structure is shown in Fig. 11a. In order to be able to observe the switching behaviour more accurately, the transmission ratio of the switch versus the input power has been depicted in Fig. 11b. We need that by doubling the input power a switching mechanism be observed in our design; therefore the triple cascade combination can be used for our design.

(a)

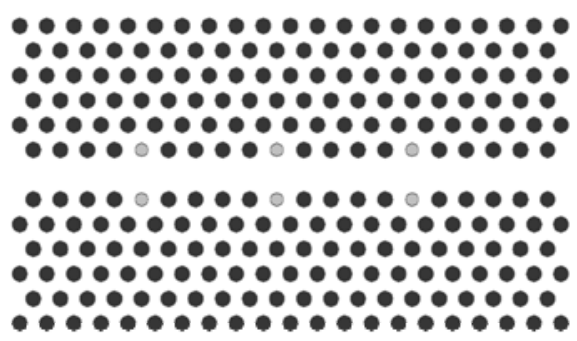

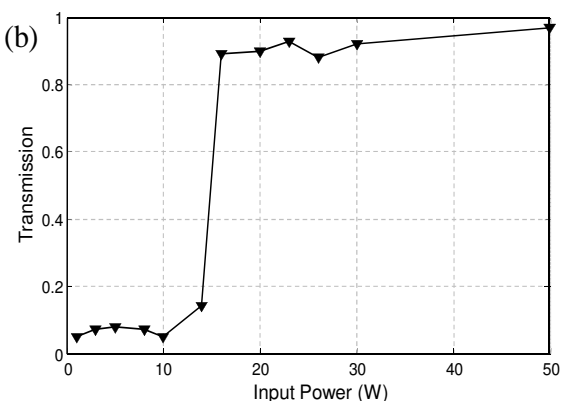

Fig. 11. (a) The limiter topology used to design an optical gate, (b) The transmission curve for the limiter in fig. 11(a).

\subsection{A PC Y-splitter}

As it was mentioned before, combination of a Y-junction and a limiter is proposed as the AND gate. Using methods presented in the literature (Wilson et al., 2003, Frandsen et. al, 2004, Yang et al, 2010, Danaie et. al, 2008) we have optimized the Y-splitter. A simple Yjunction and the optimized junction are shown in Fig. 12a and Fig. 12b respectively. By introducing modifications to the structure of the bends and branches the transmission spectrum can be improved (Wilson et al., 2003, Frandsen et. al, 2004, Yang et al., 2010). There have been some detailed topologies reported in literature which enhance the bandwidth (Borel et al., 2005, Têtu et al., 2005). (Yang et al., 2010) used a triangular lattice of dielectric rods to design a Y-Branch. They showed that the Y-branch can be treated as a cavity that couples with the input and output waveguides.

In (Yang et al., 2010) to enhance the transmittance, additional rods are added to the junction area and the corner rods are displaced. The movement of the corner rods increases the volume of their cavity and make the cavity mode resonant with the waveguide modes. It is shown in (Yang et al., 2010) that using the coupled mode theory the reflection coefficient can be expressed as (31). 


$$
R=\left|\frac{-j\left(\omega-\omega_{0}\right)+\frac{1}{\tau_{1}}-\frac{1}{\tau_{2}}-\frac{1}{\tau_{3}}}{j\left(\omega-\omega_{0}\right)+\frac{1}{\tau_{1}}+\frac{1}{\tau_{2}}+\frac{1}{\tau_{3}}}\right|^{2},
$$

where, $\omega_{0}$ is the resonance frequency, $\tau_{i}$ is the time constant regarding the amplitude decay of the resonance into the $i^{\text {th }}$ port. It is seen that if the (32) is satisfied, the reflection will be zero for $\omega=\omega_{0}$.

$$
\frac{1}{\tau_{1}}=\frac{1}{\tau_{2}}+\frac{1}{\tau_{3}}
$$

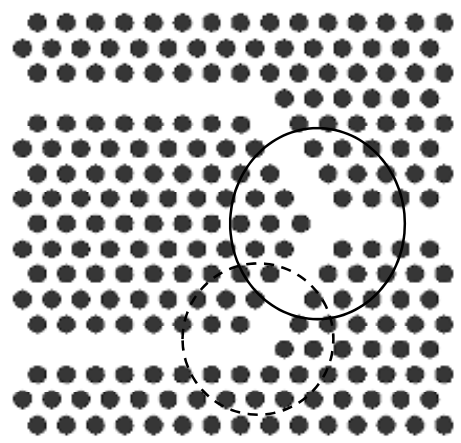

(a)

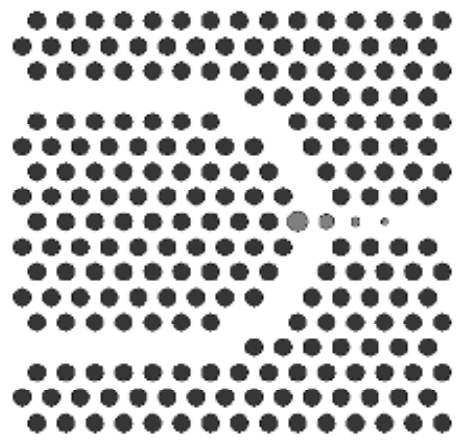

(b)

Fig. 12. (a) A simple photonic crystal Y-splitter consisting of a Y- junction (solid line) and two 60-degree bends (dashed line) (b) a Y-junction optimized to decrease the input reflection. The grey hole have radii equal to $0.4 \mathrm{a}, 0.31 \mathrm{a}, 0.15 \mathrm{a}, 0.11 \mathrm{a}$ respectively from left to right.

We already know that for our junction due to symmetry $\tau_{2}=\tau_{3}$; therefore the mentioned condition can be simplified as: $\tau_{2}=2 \tau_{1}$. It means that the decay rate of resonance to the output ports should be half the decay rate of resonance to the input port. In a simple Yjunction all the three time constants are equal. The tapered structure of the holes inserted in our proposed design, improves the coupling strength between the resonator and the input waveguide. It results in the reduction of $\tau_{1}$. If the tapering is designed so that $\tau_{1}$ is reduced to half $\tau_{2}$, then the zero reflection condition can be satisfied. Numerical optimization was used to design such a tapering.

\subsection{Design of a PC AND gate}

In order to design a PC AND gate the structure depicted in Fig. 13 is proposed. It is comprised of the proposed Y-junction in Fig. 12b and the sharp limiter in Fig. 11a. Each signal is assumed to have a power equal to $10 \mathrm{~W}$. The junction section adds the signals together. 


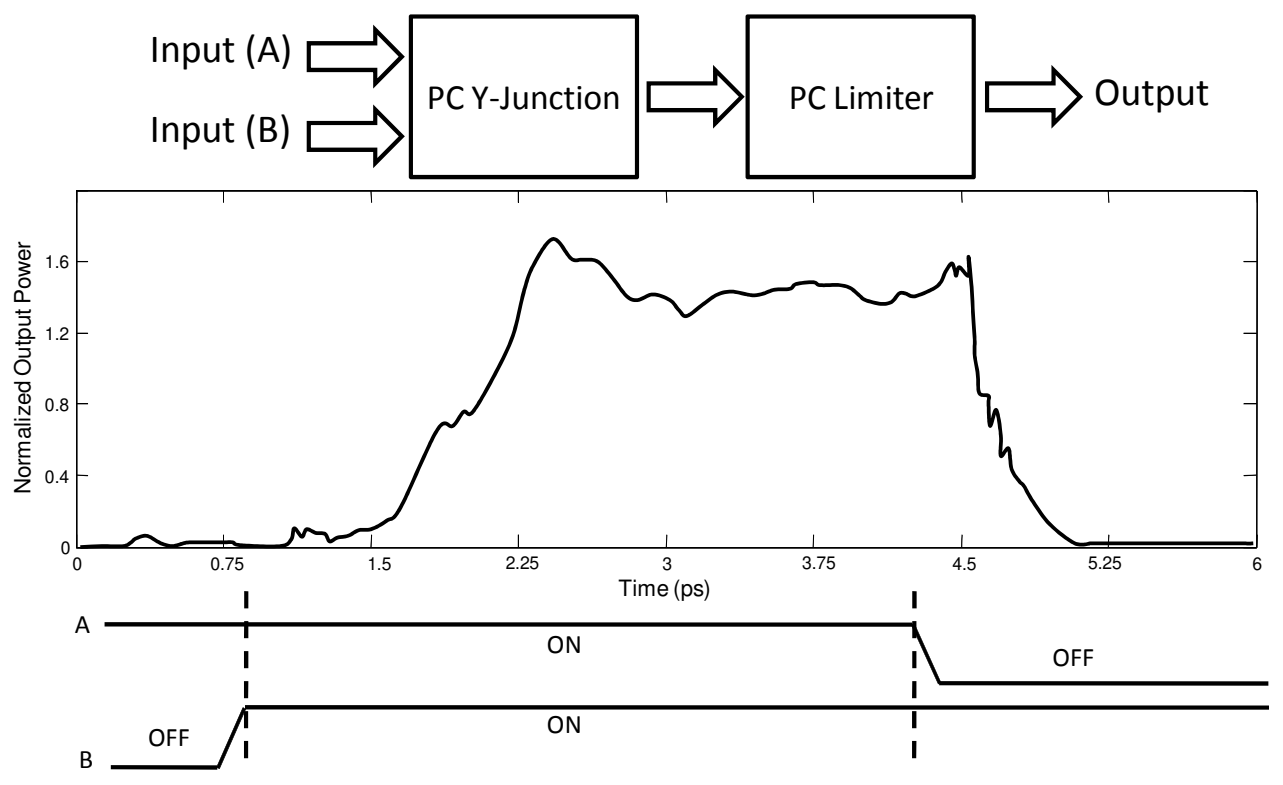

Fig. 13. The structure for the proposed AND gate.

If only one signal is sent into the junction the power will be bellow the limiter threshold and the signal will not be able to reach the output port. However when the two signals are present, the output power of the splitter will be high enough to trigger the switching mechanism of the limiter. Therefore the signal will be directed to the output port. In order to have a better insight on the performance of the device; the time domain response is also depicted. First it is assumed that only one of the input signals is ON. Then both signals are turned ON for a while and one of them is turned OFF afterwards. The normalised output power is measured for each case. It can be seen that the transition time is less than 1ps.

\subsection{Implementation issues}

Many researchers have worked on methods to efficiently couple an optical fiber to a photonic crystal waveguide. The issue of designing a coupling scheme becomes more important when photonic crystal waveguides with low group velocities are used. However, in our case since the group velocity of the W1 waveguides in inputs and outputs is not much low, the coupling problem to the W1 waveguides does not seem to be acute. In order to design the input and output couplers for the AND gate, the same efficient coupling structure implemented in (Ayre et al., 2005) can be used. The mentioned paper suggests a wideband Y-splitter in a GaAs substrate for TE modes. A $5 \mu \mathrm{m}$ ridge waveguide is coupled to the photonic crystal waveguide with period $=430 \mathrm{~nm}$ and $36.2 \%$ air filling factor (corresponding to a radius over period ( $\mathrm{r} / \mathrm{a}$ ) ratio of 0.30 ) via a $9 \mu \mathrm{m}$ injector tapering to provide a bandwidth of the order of $300 \mathrm{~nm}$.

Due to nearly exact same prameters, for the structure proposed as the AND gate and the Ysplitter in (Ayre et al., 2005), the fabrication procedure described in (Ayre et al., 2005) can 
also be employed for design and implementation of the gate. The AlGaAs/GaAs heterostructure can be grown by metal-organic chemical vapor-phase deposition (MOCVD); where a $300 \mathrm{~nm}$ silicon oxide is deposited over the wafer using plasma enhanced chemical vapor-phase deposition (PECVD), followed by a 200-nm-thick layer of PMMA as electronbeam resist. The pattern is then transferred into the oxide hard mask via reactive ion etching (RIE) using a fluorine process. The deep etching of the PC holes can be achieved using chemically assisted ion beam etching (CAIBE) in a Chlorine-Argon process (Ayre et al., 2005). The holes would thus be approximately $605 \mathrm{~nm}$ deep. The input wavelength is assumed 1550nm which can be obtained using an Erbium doped fiber laser which results in the device size to be approximately equal to $5.95 \mu \mathrm{m} \times 12.90 \mu \mathrm{m}$.

\section{References}

Andalib, P.; Granpayeh, N. (2009). All-optical ultra-compact photonic crystal NOR gate based on nonlinear ring resonators, J. of Optics A: Pure and Applied Optics, vol. 11, no. 8, (Aug. 2009) 08523.

Ayre, M.; Karle, T. J.; Wu, L.; Davies, T.; Krauss, T. F. (2005) Experimental verification of numerically optimized photonic crystal injector, Y-splitter, and bend, IEEE J. Selected Areas in Communications, vol. 23, no. 7 (Jul. 2005) pp. 1390-1395.

Bai, J.; Wang, J.; Jiang, J.; Chen, X.; Li, H.; Qiu, Y.; Qiang, Z. (2009). Photonic crystal NOT and NOR gates based on a single compact photonic crystal ring resonator, Applied Optics, vol. 48, no. 36 (Dec. 2009) pp.6923-6927.

Borel, P.I.; Frandsen, L.H.; Harpøth, A.; Kristensen, M.; Jensen, J. S.; Sigmund, O. (2005). Topology optimised broadband photonic crystal Y-splitter, IEE Electron. Let., vol. 41, no. 2 (Jan. 2005) pp. 69-71.

Boyd, R. W. (2003) Nonlinear Optics, Academic Press, San Diego, USA, ISBN: 0121216829.

Bravo-Abad, J.; Fan, S.; Johnson, S. G.; Joannopoulos, J. D.; Solja`cic', M. (2007). Modeling nonlinear optical phenomena in nanophotonics, J. of Lightwave Technol., vol. 25, no. 9, (Sep. 2007) pp. 2539-2546.

Centeno, E.; Felbacq, D; (2000). Optical bistability in finite-size nonlinear bidimensional photonic crystals doped by a microcavity, Phys. Rev. B, Condens. Matter, vol. 62, no. 12, (Sep. 2000) pp. R7683-R7686.

Cuesta-Soto, F; Martinez, A.; Garcia, J.; Ramos, F.; Sanchis, P.; Blasco, J.; Marti, J. (2004). All-optical switching structure based on a photonic crystal directional coupler, Optics Express, vol. 12, no. 1, (Jan. 2004) pp. 161-167.

Cuesta-Soto, F; Martinez, A; Garcia, J; Ramos, F; Sanchis, P; Blasco, J; Marti, J. (2004). Alloptical switching structure based on a photonic crystal directional coupler, Optics Express, vol. 12, no. 1, (Dec. 2003) pp. 161-167.

Danaie, M.; Attari, A. R.; Mirsalehi, M. M.; Naseh, S. (2008). Design of a high efficiency wide-band $60^{\circ}$ bend for TE polarization, Photonics and Nanostructures: Fundamentals and Applications, vol. 6, no. 3-4, (Dec. 2008) pp. 188-193.

Danaie, M., Kaatuzian, H. (2011). Bandwidth Improvement for a Photonic Crystal Optical Ysplitter, Journal of the Optical Society of Korea, vol. 15, no. 3 (Sep. 2011) pp. 283288.

Danaie, M.; Kaatuzian, H. (2011). Improvement of power coupling in a nonlinear photonic crystal directional coupler switch, Photonics and Nanostructures - Fundamentals and Applications, vol. 9, no. 1 (Feb. 2011) pp. 70-81. 
Fan, S. (2002). Sharp asymmetric line shapes in side-coupled waveguide-cavity systems, Appl. Phys. Lett., vol. 80, no. 6, (Feb. 2002) pp. 908-910.

Frandsen, L. H.; Borel, P. I.; Zhuang, Y. X.; Harpøth, A.; Thorhauge, M.; Kristensen, M. (2004). Ultralow-loss 3dB photonic crystal waveguide splitter, Opt. Lett., vol. 29, no. 14 (Jul. 2004) pp. 1623-1625.

Franken, P. A.; Hill, A. E.; Peters, C. W.; Weinreich, G. (1961). Generation of optical harmonics, Phys. Rev. Lett., vol. 7, no. 4, (Aug. 1961) pp. 118-199.

Fushman, I.; Englud, D.; Faraon, A.; Stoltz, N.; Petroff, P.; Vuckovic, J. (2008) Controlled phase shifts with a single quantum dot, Science, vol. 320, no. 5877 (May 2008) pp. 769-772.

John, S. (1987). Strong localization of photons in certain disordered dielectric superlattices, Phys. Rev. Lett., vol. 58, no. 23, (Jun. 1987) pp. 2486-2489.

Joseph, R. M.; Taflov, A. (1997). FDTD Maxwell's equations models for nonlinear electrodynamics and optics, IEEE Tran. on Anten. and Prop., vol. 45, no. 3, (Mar 1997) pp. 364-374.

Kaatuzian, H. (2008) photonics, vol. 1, 2nd edition (in Persian), Amirkabir University Press, Tehran, Iran, ISBN: 9644632710.

Liu, Q; Ouyang, Z; Wu, C. J.; Liu, C. P.; Wang, J. C. (2008). All-optical half adder based on cross structures in two-dimensional photonic crystals, Optics Express, vol. 16, no. 26. (Nov. 2008) pp.18992-19000.

Locatelli, A; Modotto, D; Paloschi, D; Angelis, C. D. (2004). All optical switching in ultrashort photonic crystal couplers, Optics Commun., vol. 237, (March) pp. 97-102.

Maiman, T. H. (1960). Stimulated optical radiation in ruby. Nature, vol. 187 (4736): (Aug. 1960) pp. 493-494.

Mingaleev, S. F.; Kivshar, Y. S. (2002). Nonlinear transmission and light localization in photonic-crystal waveguides, J. Opt. Soc. Amer. A, Opt. Image Sci., vol. 19, no. 9, (Sep. 2002) pp. 2241-2249, ISSN: 0740-3224.

Nagpal, Y.; Sinha, R. K. (2004) Modelling of photonic band gap waveguide couplers, Microwave and Opt. Technol., Lett., vol. 43, no. 1 (Oct. 2004) pp. 47-50.

Nakamura, H.; Sugimoto, Y.; Kanamoto, K.; Ikeda, N.; Tanaka, Y.; Nakamura, Y.; Ohkouchi, S.; Watanabe, Y.; Inoue, K.; Ishikawa, H.; Asakawa, K. (2004) Ultrafast photonic crystal/quantum dot all-optical switch for future photonic networks, Optics Express, vol. 12, no. 26 (Dec. 2004) pp. 6606-6614.

Rahmati, A. T.; Granpayeh, N. (2010). Design and simulation of a switch based on nonlinear directional coupler, Optik -Internat. J. for Light and Electron. Optics, vol. 121, no. 18, (Oct. 2010) pp. 1631-1634.

Saleh, B. E. A.; Teich, M. C. (1991). Fundamentals of Photonics, John Wiley \& Sons, New York, USA, ISBN: 0471839655.

Scholz, S.; Hess, O.; R"uhle, R. (1998). Dynamic cross-waveguide optical switching with a nonlinear photonic band-gap structure, Optics Express, vol. 3, no. 1, (Jul. 1998) pp. 28-34.

Shinya, A.; Matsuo, Yosia, S; Tanabe, T.; Kuramochi, E; Sato, T.; Kakitsuka, T.; Notomi, M. (2008). All-optical on-chip bit memory based on ultra high Q InGaAsP photonic crystals, Optics Express, vol. 16, no. 23, (Nov. 2008) pp. 19382-19387. 
Solja 'cic', M.; Ibanescu, M.; Johnson, S. G.; Fink, Y.; Joannopoulos, J. D. (2002). Optimal bistable switching in nonlinear photonic crystals, Phys. Rev. E, vol. 66, no. 5, (Nov. 2002) pp. 055601R.

Solja 'cic', M.; Joannopoulos, J. D. (2004) Enhancement of nonlinear effects using photonic crystals, Nat. Mater., vol. 3, no. 4, (Apr. 2004) pp. 211-219.

Têtu, A.; Kristensen, M.; Frandsen, L.; Harpøth, A.; Borel, P.; Jensen, J.; Sigmund, O. (2005) Broadband topology-optimized photonic crystal components for both TE and TM polarizations, Optics Express, vol. 13, no. 21 (Oct. 2005) pp. 8606-8611.

Weinberger, P. (2008). John Kerr and his Effects Found in 1877 and 1878, Philosophical Magazine Letters, vol. 88, no. 12, (Dec 2008) pp.897-907.

Wilson, R.; Karle, T. J.; Moerman, I.; Krauss, T. F. (2003). Efficient photonic crystal Yjunctions, J. Opt. A: Pure Appl. Opt., vol. 5, no. 4 (Jul. 2003) pp.S76-S80.

Yablonovitch, E. (1987). Inhibited spontaneous emission in solid-state physics and electronics, Phys. Rev. Lett., vol. 58, no. 20, (May 1987) pp. 2059-2062.

Yamamoto, N.; Ogawa, T; Komori, K. (2006). Photonic crystal directional coupler switch with small switching length and wide bandwidth, Optics. Express, vol. 14 (Feb. 2006) pp. 1223-1229.

Yang, W.; Chen, X.; Shi, X.; Lu, W. (2010) Design of high transmission Y-junction in photonic crystal waveguides, Physica B: Condensed Matter., vol. 405, no. 7 (Apr. 2010) pp. 1832-1835.

Yanik, M. F.; Fan, S.; Solja 'cic', M. (2003). High-contrast all-optical switching in photonic crystal microcavites, App. Phys. Lett., vol. 83, no. 14, (Oct. 2003), pp. 2739-2741.

Zhu, Z.-H.; Ye, W.-M.; Ji, J.-R.; Yuan, X.-D. ; Zen, C. (2006). High-contrast light-by-light switching and AND gate based on nonlinear photonic crystals, Optics Express, vol.14, no. 5, (Mar. 2006)pp. 1783-1788.

Zimmermann, J.; Kamp, M.; Forchel, A.; Marz, R. (2004). Photonic crystal waveguide directional couplers as wavelength selective optical filters, Opt. Communications, vol. 230 no. 4-6, (Feb. 2004) pp. 387-392. 


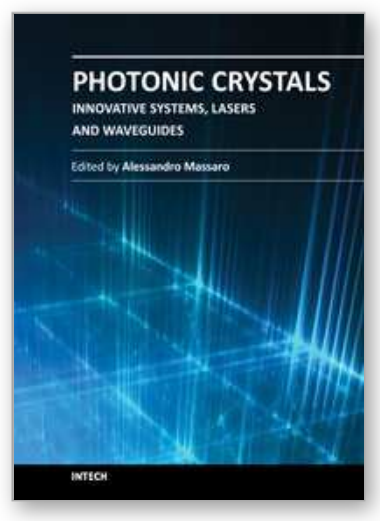

\author{
Photonic Crystals - Innovative Systems, Lasers and Waveguides \\ Edited by Dr. Alessandro Massaro
}

ISBN 978-953-51-0416-2

Hard cover, 348 pages

Publisher InTech

Published online 30, March, 2012

Published in print edition March, 2012

The second volume of the book concerns the characterization approach of photonic crystals, photonic crystal lasers, photonic crystal waveguides and plasmonics including the introduction of innovative systems and materials. Photonic crystal materials promises to enable all-optical computer circuits and could also be used to make ultra low-power light sources. Researchers have studied lasers from microscopic cavities in photonic crystals that act as reflectors to intensify the collisions between photons and atoms that lead to lazing, but these lasers have been optically-pumped, meaning they are driven by other lasers. Moreover, the physical principles behind the phenomenon of slow light in photonic crystal waveguides, as well as their practical limitations, are discussed. This includes the nature of slow light propagation, its bandwidth limitation, coupling of modes and particular kind terminating photonic crystals with metal surfaces allowing to propagate in surface plasmon-polariton waves. The goal of the second volume is to provide an overview about the listed issues.

\title{
How to reference
}

In order to correctly reference this scholarly work, feel free to copy and paste the following:

Mohammad Danaie and Hassan Kaatuzian (2012). Employing Optical Nonlinearity in Photonic Crystals: A Step Towards All-Optical Logic Gates, Photonic Crystals - Innovative Systems, Lasers and Waveguides, Dr. Alessandro Massaro (Ed.), ISBN: 978-953-51-0416-2, InTech, Available from: http://www.intechopen.com/books/photonic-crystals-innovative-systems-lasers-and-waveguides/employingoptical-nonlinearity-in-photonic-crystals-a-step-towards-all-optical-logic-gates

\section{INTECH}

open science | open minds

\section{InTech Europe}

University Campus STeP Ri

Slavka Krautzeka 83/A

51000 Rijeka, Croatia

Phone: +385 (51) 770447

Fax: +385 (51) 686166

www.intechopen.com

\section{InTech China}

Unit 405, Office Block, Hotel Equatorial Shanghai

No.65, Yan An Road (West), Shanghai, 200040, China

中国上海市延安西路65号上海国际贵都大饭店办公楼 405 单元

Phone: +86-21-62489820

Fax: $+86-21-62489821$ 
(C) 2012 The Author(s). Licensee IntechOpen. This is an open access article distributed under the terms of the Creative Commons Attribution 3.0 License, which permits unrestricted use, distribution, and reproduction in any medium, provided the original work is properly cited. 